\title{
Quasi-Dammann grating with proportional intensity of array spots for surface hardening of metal
}

\author{
LI ShaoXia ${ }^{1,2}$, TAN QiaoFeng ${ }^{2}$, YU Gang ${ }^{1 *}$, WANG HengHai ${ }^{1,3} \&$ JIN GuoFan ${ }^{2}$ \\ ${ }^{1}$ Key Laboratory of Mechanics in Advanced Manufacturing, Institute of Mechanics, Chinese Academy of Sciences, Beijing 100190, China; \\ ${ }^{2}$ State Key Laboratory of Precision Measurement Technology and Instruments, Tsinghua University, Beijing 100084, China; \\ ${ }^{3}$ The 14th Research Institute, China Electronics Technology Group Corporation, Nanjing 210039, China
}

Received April 19, 2010; accepted July 30, 2010; published online October 28, 2010

\begin{abstract}
For surface hardening of metal, a quasi-Dammann grating (QDG) is proposed and fabricated to generate array spots with proportional-intensity distribution. To get uniformly hardened band distribution and improve the wear resistance of the sample surface, a three-order QDG is designed to produce array spots with enhanced intensity in the edge. The design and fabrication of the QDG are described in detail. The surface profile of the fabricated grating was measured, which shows that the fabrication error is less than $2 \%$. The laser beam intensity distribution shaped by the QDG was tested by a laser beam analyzer to verify the validity of the QDG. The application of the QDG in the laser surface hardening of metal was experimentally investigated, and the results show that the hardness distribution of the modified layer and the wear resistance of the sample surface are improved significantly by using the QDG.
\end{abstract}

quasi Dammann grating, beam shaping, laser surface hardening, diffraction

PACS: 42.25.Fx, 41.85.Ct, 42.60.Jf, 42.62.Cf

\section{Introduction}

Laser surface hardening of metal has been used in industrial applications, as it prevents advance abrasion or cracks of the surface. And the laser beam used in laser surface hardening of metal processes is always the focused or defocused original Gaussian spots [1]. In this way, the laser transformation hardening band is a crescent with deep center and edge gradually changing to shallow, which is different from the uniform hardened band one hopes. To get uniformly hardened band, the laser beam is always transformed to rectangle spot with constant intensity distribution [2,3]. Recently we [4] have reported a method of laser surface modification of ductile iron using laser beam with equal-intensity array spots. In that research, traditional Dammann Grating [5] was used to transform the Gaussion laser beam to equal-

*Corresponding author (email: gyu@imech.ac.cn) intensity array spots. The application of the Dammann gratings in surface modification made the ductile iron surface show periodic gradient hardness distribution [6], and the wear resistance of ductile iron has been improved [4]. But in all these researches the hardened band is still crescent-like shape. The theory and the experiments indicate that, even if the specimen is a simple flat plate work piece, to get uniform hardened band, a laser beam with intensity blow-up in the edge of the spot is needed.

The purpose of this paper is to employ a quasi-Dammann grating (QDG) [7] to transform the laser beam into array spots with edge intensity enhanced in the far field. And hence, get uniform shaped hardened band and better wear resistance of the sample surface hardened.

\section{Grating design and fabrication}

The QDG is a kind of improved Dammann grating that can 
produce high efficiency array pattern with proportionalintensity orders in the far field. In ref. [7], parameters for characterization of QDG, such as the diffractive order, the uniformity and diffractive efficiency have been defined.

To get an array laser spot with intensity enhanced in the edge for the application of laser surface hardening of metal, a three-order QDG with intensity proportion of $I_{(0)}: I_{(1)}: I_{(2)}=1: 2: 3$ from zero order to the second order was designed and fabricated as an example. According to the definition of merit function of QDG, the merit function can be expressed as

$$
\begin{aligned}
E^{2}= & \alpha\left\{\left[\beta_{1}\left(I_{(0)}-\eta_{E} \hat{I_{(0)}}\right)\right]^{2}+\left[\beta_{2}\left(I_{(1)}-\eta_{E} \hat{I_{(1)}}\right)\right]^{2}\right. \\
& \left.+\left[\beta_{3}\left(I_{(2)}-\eta_{E} \hat{I_{(2)}}\right)\right]^{2}\right\}+(1-\alpha)\left(1-\eta_{E}\right)^{2},
\end{aligned}
$$

where $\alpha \subseteq[0,1]$ is the weight coefficient, $\eta_{E}$ is the diffraction efficiency of the QDG, $\hat{I}_{(0)}, \hat{I}_{(1)}, \hat{I}_{(2)}$ are respectively the ideal intensities of the order 0,1 and 2 , and $\hat{I}_{(0)}, \hat{I}_{(1)}, \hat{I}_{(2)}$ are respectively the intensities of order 0,1 , and 2. $\beta_{1}, \beta_{2}, \beta_{3}$ are the weight coefficients of the orders, and according to the intensity distribution of $I_{(0)}: I_{(1)}: I_{(2)}=k_{1}: k_{2}: k_{3}=1: 2: 3$, then $\beta_{1}, \beta_{2}, \beta_{3}$ should have the relationship of $\beta_{1}: \beta_{2}: \beta_{3}=1 / k_{1}: 1 / 2 k_{2}: 1 / 2 k_{3}=$ $1: 1 / 4: 1 / 6$.

The simulated annealing (SA) algorithm [8-10] is adopted to optimize the QDG. The designed result of the three-order QDG with $I_{(0)}: I_{(1)}: I_{(2)}=1: 2: 3$ is shown in Figure 1. The sampling number of one period of the three-order QDG is 16×16 (Figure 1(a)). Figure 1(b) is the designed intensity distribution of the QDG. The in-order uniformities of the first and second order are $U_{o, 1}=1.4 \%$ and $U_{o, 2}=3.25 \%$, respectively. The in-order uniformity of the zero order is insignificant because there is only one point in this order. The proportional uniformity is $U_{p}=1.2: 1.1: 1.0$, and the diffraction efficiency is

$$
\eta_{E}=\sum_{\substack{m=1 \\ m=0}}^{M} I_{m n} / I_{\text {total }}=70.9 \% .
$$

The very large-scale integration (VLSI) technique [11] was adopted in our experiment to fabricate the QDG. To be agree with the size of the laser equipment, the diameter of the element fabricated was $50 \mathrm{~mm}$, and the period was $d=250 \mu \mathrm{m}$. With wavelength $1.064 \mu \mathrm{m}$, and focal length of the lens $f=150 \mathrm{~mm}$, the interval of the adjacent spot of the shaped beam was $\Delta=\lambda f / d \approx 0.6 \mathrm{~mm}$. The diameter of a single spot of the shaped focal spot was $D=0.45 \mathrm{~mm}$. The first step was to make the mask with electron-beam writing equipment. A thin layer of photo-resist was spun onto a glass substrate. With ordinary microelectronic lithography technology, the mask pattern was transformed into a photo-resist layer upon a glass substrate. The inductive coupled plasma technique was used to transfer the photo-resist pattern onto the substrate. The refractive index of the glass that was used at a wavelength of $1.064 \mathrm{~mm}$ was $n=1.507$, and the thickness corresponding to the phase difference $\pi$ was $1046 \mathrm{~nm}$. The surface profile of the grating measured with Dektak8, which is an advanced surface texture measuring system, is shown in Figure 2, clearly shows that the average depth of this surface-relief element is $1064.4 \mathrm{~nm}$, slightly deviating from the desired value. The fabrication error is less than $2 \%$.

\section{Experimental results}

The experimental optical system is shown in Figure 3. A collimated laser beam illuminates the QDG located in front of the lens. The surface to be hardened is put in the rear focal plane, where the shaped intensity distribution of the laser beam is obtained. A collimated $1.064 \mu \mathrm{m}$ wavelength
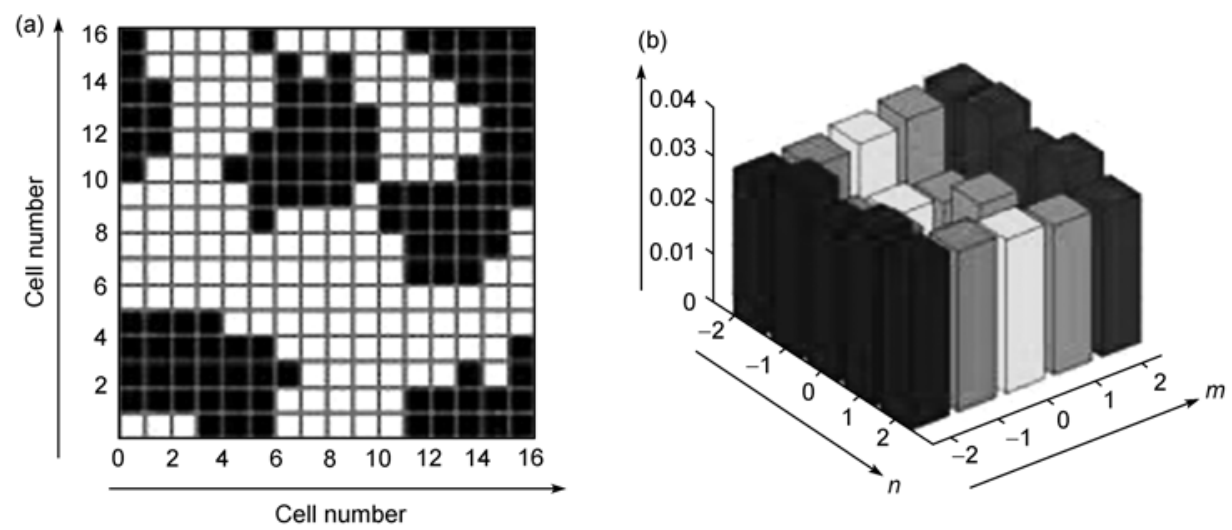

Figure 1 Designed result of the three-order QDG with proportional intensity (1:2:3 from order 0 to order 2). (a) Phase distribution of one period; (b) output intensity profile. 


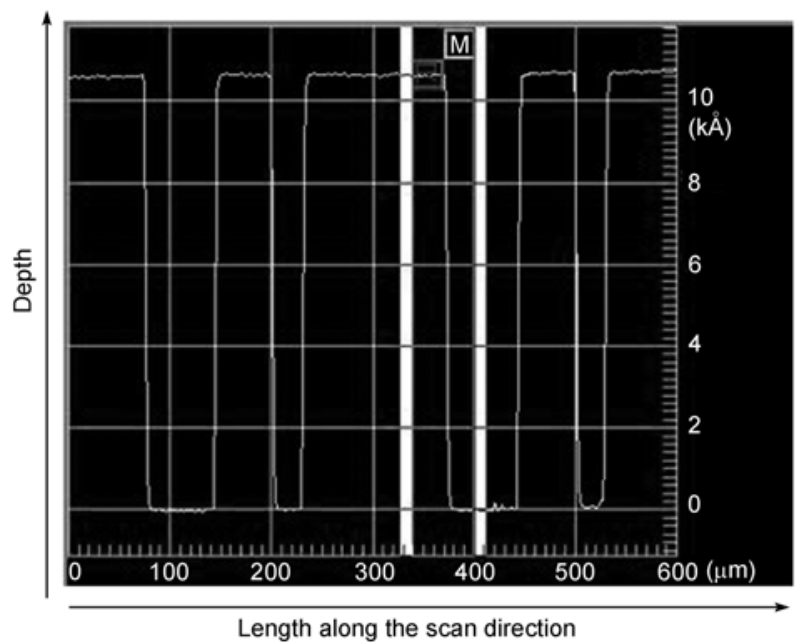

Figure 2 Surface profile of the fabricated three-order QDG with proportional intensity.

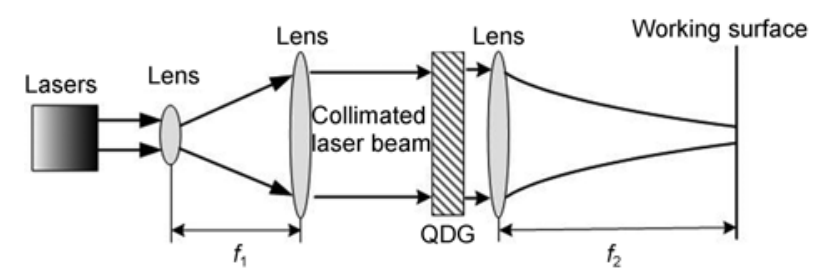

Figure 3 Experimental arrangement for laser beam shaping and application.

HLD1001.5 laser is used as the light source with a lens of $150 \mathrm{~mm}$ focal length. The three-order array pattern with an intensity proportion of 1:2:3 from the zero order to the second order captured by a laser beam analyzer in the rear focal plane of the lens is shown in Figure 4. The slightly lower efficiency and worse uniformity might be due to the surface roughness of the fabricated element, the phase and position errors, and the dark noise of the laser beam analyzer. Nevertheless, the experimental results verify the validity of the QDG with intensity proportion of 1:2:3.

The Q235 steel, with nominal chemical composition (at\%) of $0.14-0.22 \mathrm{C}, 0.3-0.65 \mathrm{Mn}, 0.30 \mathrm{Si}, 0.050 \mathrm{~S}, 0.045 \mathrm{P}$, and balance $\mathrm{Fe}$, was selected as the tested material because it is one of the most common materials used in industry. The steel specimens, $100 \mathrm{~mm} \times 50 \mathrm{~mm} \times 20 \mathrm{~mm}$ in size, were laser hardened by a HLD1001.5D Nd:YAG laser equipped with the designed QDG. To give a comparison, a traditional Dammann grating with $5 \times 5$ equal intensity array spots was also introduced to do the surface hardening of the sample. The laser processing parameters were as follows: laser output power was $1000 \mathrm{~W}$ pulse duration was $170 \mathrm{~ms}$ and 100 $\mathrm{ms}$ for the QDG with intensity distribution of 1:2:3 and for the traditional Dammann Grating with $5 \times 5$ equal-intensity array, respectively. The samples were hardened separately by the array spots with intensity distribution of $1: 2: 3$ and the $5 \times 5$ equal intensity array spots at the focal plane. To sim- plify expression, sample hardened by $5 \times 5$ equal intensity array spots is called sample $A$, and sample hardened by 1:2:3 proportional intensity array spots is called sample $B$.

To describe the hardened layer of the material, the uniformity of the hardened layer pattern can be defined as the area ratio of the hardened layer to the rectangle it occupies. Figure 5 is the hardened layer pattern of the samples. It can be clearly seen that the hardened layer pattern of the sample hardened with equal intensity $5 \times 5$ array spots is crescent-like shape. But the hardened layer pattern of the sample hardened with beam shaped by QDG is close to rectangular, which means the use of QDG does improve the uniformity of the surface hardened band.

The hardness along the layer depth of the modified material was measured, and the load was 50 GF. To analyze the hardness distribution of the layer, the hardness along the layer depth was measured along the line perpendicular to the surface via the center and 1/3 diameter of the laser spot away from the center of the action zone individually as the dashed lines shown in Figure 6. And the microhardness curves of the surface layer of sample A and sample B are shown in Figures 7 and 8, respectively. The contrast of Figures 7 and 8 shows that the microhardness curves via the center of the layers of the two samples all fluctuate from $430 \mathrm{HV}$ to $180 \mathrm{HV}$ within the depth from 50 to $550 \mu \mathrm{m}$. The curve measured via the point $1 / 3 D$ away from the center of Sample A drops rapidly from $300 \mathrm{HV}$ to $160 \mathrm{HV}$ in the depth of $300 \mu \mathrm{m}$ to $500 \mu \mathrm{m}$. While the curve measured via the point $1 / 3 \mathrm{D}$ away from the center of sample $\mathrm{B}$ have the similar fluctuation direction with the curve measured via the center, especially in the depth 300 to $450 \mu \mathrm{m}$ the hardness fluctuate among a small interval of $200 \mathrm{HV}$ to $250 \mathrm{HV}$, which means the microhardness of the layer of sample B is more homogeneous than that of sample A. This result agrees with the analysis of the hardened layer pattern uniformity degree. On the other hand, from Figure 8 one can see that at the same layer depth the hardness measured via the point $1 / 3 D$ away from the center is lower than that measured via the center, this means that the intensity proportion of 1:2:3 is not the best intensity distribution.

Oil-lubricated sliding wear testing was taken to compare the wear resistance property of the base metal surface without treating, the surface treated by $5 \times 5$ uniform array spots and that treated by array spots with $1: 2: 3$ proportional intensity distributions. The experiments were performed under ring rotary speed of $40 \mathrm{rpm}$, load of $2000 \mathrm{~N}$, time duration of two hours, and at room temperature. As can be seen in Figure 9, the mass loss of the base material, Sample A and Sample B is $1.78 \mathrm{mg}, 0.35 \mathrm{mg}$ and $0.21 \mathrm{mg}$, respectively. The results indicate that the wear resisting property of the samples is highly improved by the shaping laser beams. Moreover the laser beam with 1:2:3 intensity distribution array spots is better than the $5 \times 5$ equal intensity array spots in improving the wear resisting property of the samples. The result implies that the use of QDG can 


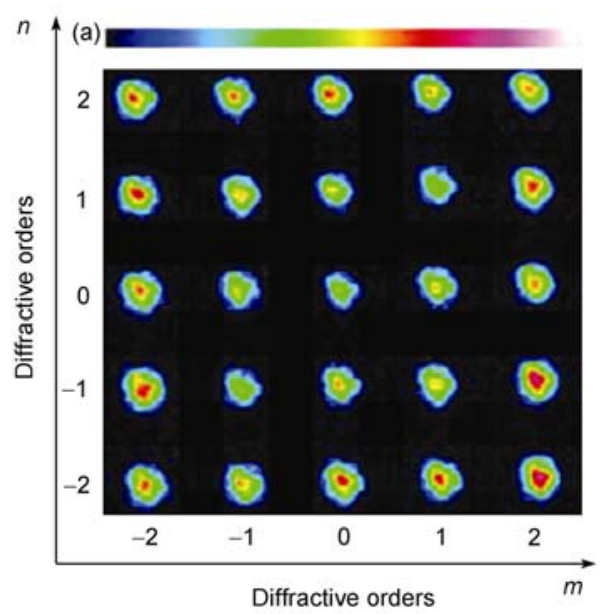

(b)

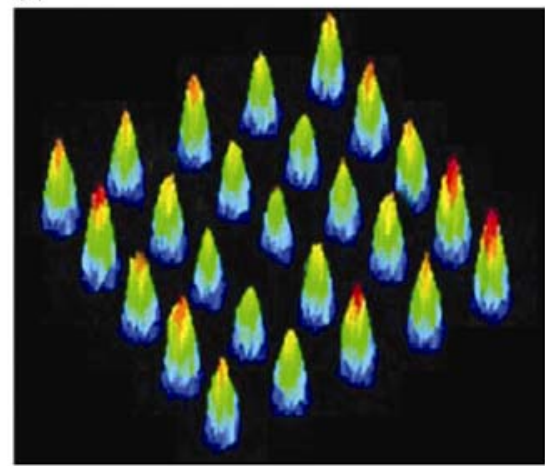

Figure 4 Experimental images of the fabricated three-order QDG with proportional intensity. 2D laser beam distribution is represented in (a) and 3D laser beam distribution is represented in (b).

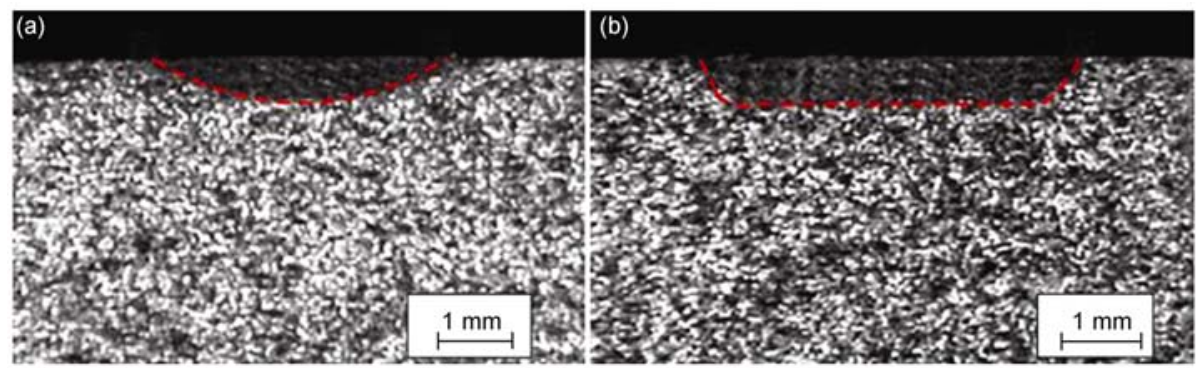

Figure 5 Microstructure of the modified layer modified by laser beam shaped by $5 \times 5$ Dammann grating (a), and laser beam shaped by the three-order QDG (b).

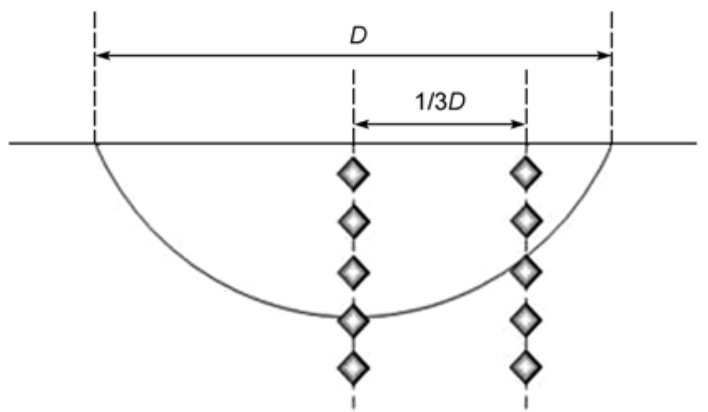

Figure 6 Microhardness testing sketch (Microhardness along the layer depth was measured at the center of the layers and at the $1 / 3 D$ away from the center of the layer).

improve the wear resistance of the metal parts under high impact load bearing conditions and, hence, enhances their service life time.

\section{Conclusion}

An array of laser beam pattern with intensity enhanced in the edge was achieved by using QDG for the application of laser surface hardening of metal. As to different materials

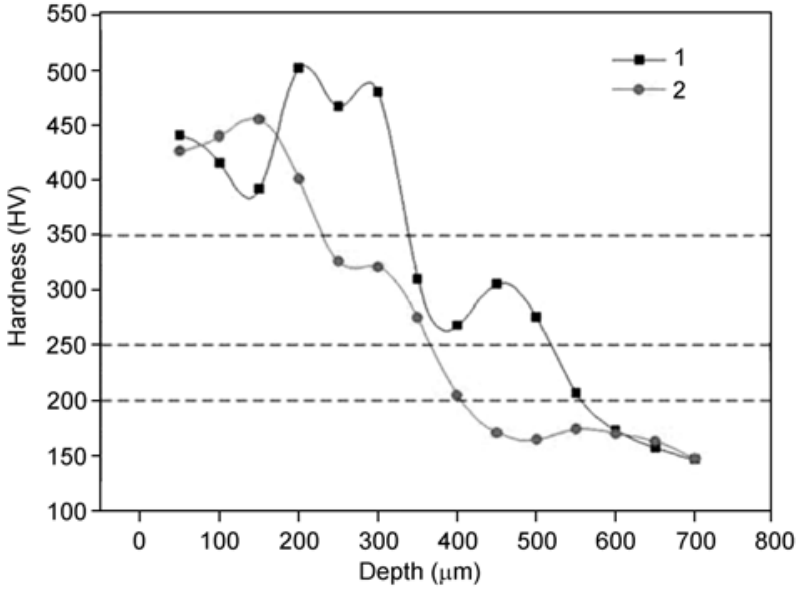

Figure 7 Microhardness distribution along the layer depth of the sample A. Line 1: Microhardness curve measured at the center of the layers; Line 2: Microhardness curve measured at the $1 / 3 D$ away from the center of the layer.

and hardness distribution requirements, the QDG with corresponding orders and order intensity proportions can be prepared. This method is flexible and low cost for beam shaping in laser surface hardening of metal. The QDG designed and fabricated in this paper can transform Nd:YAG 


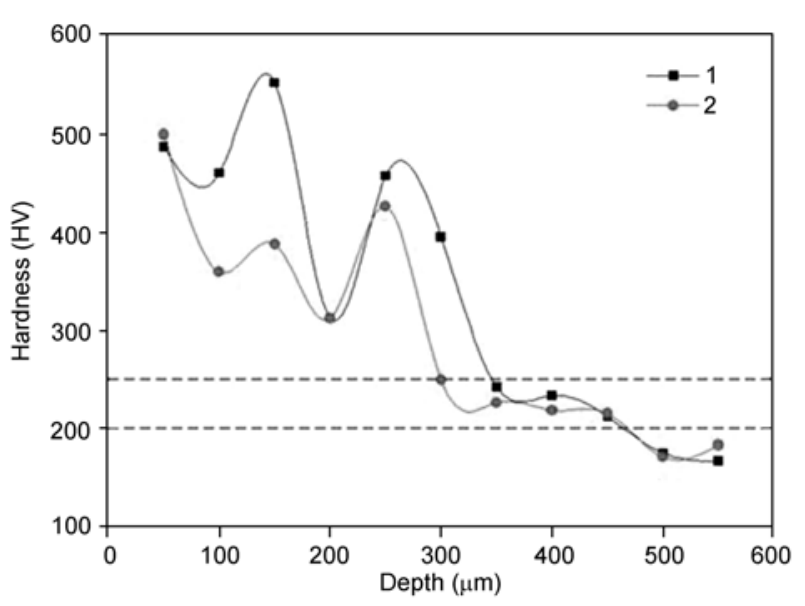

Figure 8 Microhardness distribution along the layer depth of the sample B. Line 1: Microhardness curve measured at the center of the layers; Line 2: Microhardness curve measured at the $1 / 3 D$ away from the center of the layer.

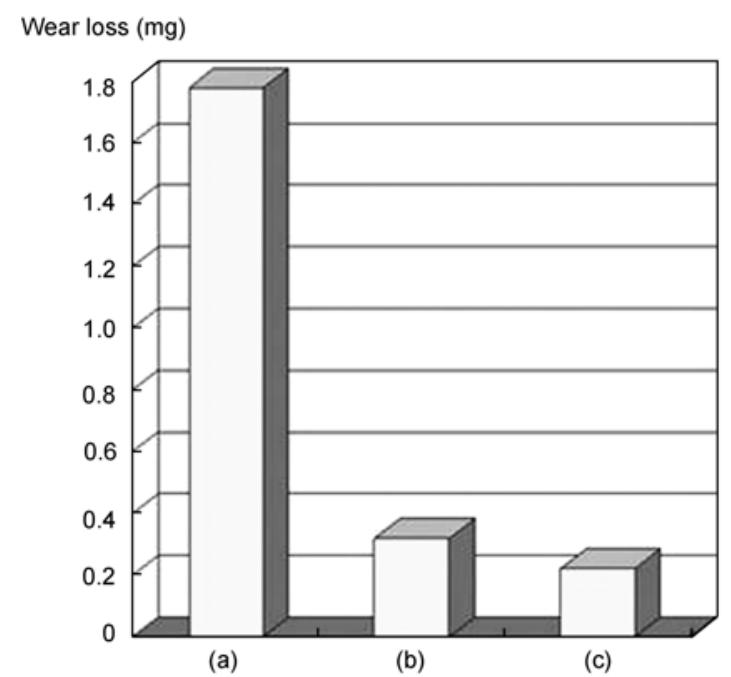

Figure 9 Mass loss of the base material (a), sample treated by laser beam with $5 \times 5$ equal intensity array spots (b), and sample treated by laser beam shaped by QDG (c). laser beam into array spots with intensity proportion of $1: 2: 3$ from the zero order to the second order. And it is well applied in laser surface hardening of Q235 metal. The experiments show that the application of the QDG in surface hardening made the Q235 metal surface have more uniform hardened band, resulting in more uniform hardness distribution and better wear resistance.

This work was supported by the National Natural Science Foundation of China (Grant No. 10832011) and the National Science Foundation for Post-doctoral Scientists of China (Grant No. 20100470139).

1 Roy A, Manna I. Mathematical modeling of localized melting around graphite nodules during laser surface hardening of austempered ductile iron. Opt Lasers Eng, 2000, 34: 369-383

2 Taghizadeh M R, Blair P, Ballüder K, et al. Design and fabrication of diffractive elements for laser material processing applications. Opt Lasers Eng, 2000, 34: 289-307

3 Li J, Li X, Chen Q, et al. Fast Calculation of thermal action of actual beam during the laser heat treatment. Chin J Mater Res, 1998, 12: 262-266

4 Li S, Yu G, Zheng C, et al. High power laser beam shaping by inseparable two dimensional binary-phase gratings for surface modifying of stamping dies. Opt Lasers Eng, 2008, 46(7): 509-513

5 Dammann H, Gốrter K. High-efficiency in line multiple imaging by means of multiple phase holograms. Opt Commun, 1971, 3: 312-315

6 Chen Y, Gan C, Wang L, et al. Laser surface modified ductile iron by pulsed Nd:YAG laser beam with two-dimensional array distribution. Appl Surf Sci, 2005, 245: 316-321

7 Li S, Yu G, Zheng C, et al. Quasi-Dammann grating with proportional intensity array spots. Opt Lett, 2008, 33(18): 2023-2025

8 Yoshikawa N, Itoh M, Yatagai T. Phase optimization of a kinoform by simulated annealing. Appl Opt, 1994, 33(5): 863-868

9 Kim M S, Guest C G. Simulated annealing algorithm for binary phase only filters in pattern classification. Appl Opt, 1990, 29(8): 1203-1208

10 Stephan T, Brigitte S, Thomas B. Efficient design of paraxial diffractive phase elements with descent search methods. In: Cindrich I, Lee S H, eds. Diffractive and Holographic Optics Technology II SPIE Proceedings, 1995, 2404: 40-49

11 Margaret B S. A VLSI-based microoptics technology. Microelectron Eng, 1996, 32: 369-388 